\title{
Jean-Laurent Casanova honored with the 2016 ASCI/Stanley J. Korsmeyer Award
}

$\mathbf{L}$ ast year, Jean-Laurent Casanova published a landmark article in Science demonstrating that a child had life-threatening influenza because of genetic loss of interferon regulatory factor 7 (1). This paper provided the first evidence that severe influenza could be due to mutations in genes encoding factors that are specifically required for responding to influenza infection rather than mutations that cause a general immunodeficiency. The study followed on two decades of research from Dr. Casanova's laboratory demonstrating that individual cases of severe infection can result from an underlying human genetic defect in a number of different infectious diseases. With this year's Stanley J. Korsmeyer Award, the ASCI recognizes Jean-Laurent Casanova's pioneering work in the field of human genetics of infectious diseases (Figure 1). The JCI recently had the opportunity to speak with him about the prize and his research accomplishments.

JCI: What attracted you first to a career in medicine and then in research?

Casanova: Immediately after high school, I began both a medical and a scientific curriculum, with the intention to pursue both careers together. I tailored my own physician-scientist training program at two separate universities, Paris Descartes University for the MD and the residency in pediatrics, and Paris Pierre et Marie Curie University for the MSc and the $\mathrm{PhD}$ in immunology, sometimes in the same year (the MD and MSc years) - sometimes by taking years off from one program to pursue the other (the $\mathrm{PhD}$ years and the residency). I wanted to become a physician-scientist. I knew that at the age of 18 years, and by the age of 32 I was fully trained as a pediatrician and as an immunologist. I have luckily not changed my mind since.

JCI: Your early studies focused on $\mathrm{T}$ cell receptor selection and recognition of MHC-peptide complexes. How did this line of study lead to human genetics work?

Casanova: I wanted to walk on two legs. I spent much time in pediatric intensive care during my residency, to truly understand and practice pediatrics. Likewise, I purposely did a PhD in immunology

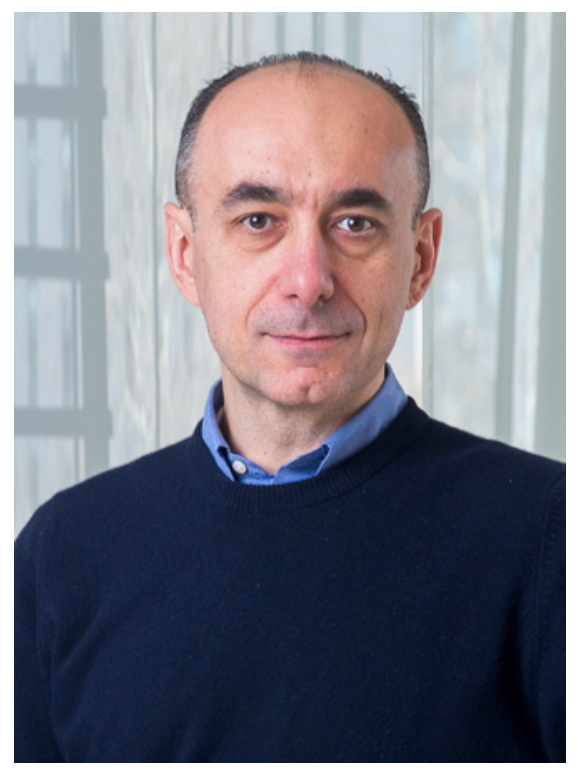

Figure 1. Jean-Laurent Casanova is the recipient of the $2016 \mathrm{ASCl} / \mathrm{Stanley} \mathrm{J.} \mathrm{Korsmeyer}$ Award in recognition for his work demonstrating that single-gene mutations can underlie life-threatening infectious diseases in otherwise healthy children and young adults. Image credit: Mario Morgado.

in a field as basic and unrelated to medicine as possible. I studied the recognition of HLA peptides embedded in $\mathrm{H}-2$ molecules by mouse cytotoxic T cells. One can hardly think of something more immunological. It was a wonderful period of my life. I was supervised by Janet Maryanski in Lausanne and by Philippe Kourilsky in Paris, while collaborating with Pedro Romero, Bernard Malissen, and Rob MacDonald. Reading Claude Bernard's introduction to the study of experimental medicine almost every day also contributed greatly to my understanding of and apprenticeship in the scientific process; there is much more to learn in Claude Bernard than in Karl Popper, especially for a biologist.

It's only after having received a strong training in basic immunology and clinical pediatrics that I decided the time had come to benefit from these two cultures and tackle a problem that would be important from both perspectives - a biological and medical problem. I focused on what I like to refer to as the infection enigma - why only a small proportion of infected children, adolescents, and young adults develop life-threatening infectious disease in the course of primary infection by almost any microbe. This problem was posed around 1910 and had been left unsolved. As a matter of fact, it was not even tackled. So my PhD did not lead me to the study of human genetics of infectious diseases, but my dual training in clinical pediatrics and basic immunology prepared me for this jump - both to see the problem and to find ways to tackle it.

JCI: Your first human genetics paper described a case report of idiopathic bacillus Calmette-Guérin (BCG) infection in a child following tuberculosis vaccination (2). What made you suspect a genetic origin?

Casanova: The lack of an alternative, plausible, and testable hypothesis, at least in my eyes 20 years ago! It is still the case today. And I think Mike Levin, who authored the companion paper, reporting a family with idiopathic environmental mycobacterial disease, felt the same (3). When a child dies of infection, whether natural (like environmental mycobacteriosis) or iatrogenic (in the case of the BCG vaccine), and there is no overt acquired immunodeficiency, I simply cannot find a better hypothesis than a hidden inborn immunodeficiency. This is logical, and I think confusion in the field of infectious diseases has come from the popular but faulty notion that an infection can kill an "immunocompetent" individual. Once you open your eyes and admit, once and for all, that death from infection is compelling evidence for immunodeficiency, 
even if you do not detect immunological signs of immunodeficiency, the rest follows logically. As always, it is the view of the whole organism that should prevail. The only physiology that matters operates at the level of the whole organism, and real physiologists have reductionist hands but a holistic mind.

JCI: As you described additional patients, including those with partial interferon- $\gamma$ receptor 1 deficiency, was it surprising that there was a relatively limited spectrum of pathogens that these individuals were susceptible to?

Casanova: This is surprising from the angle of the mouse Th1 paradigm, which assigned to IFN- $\gamma$ and IL-12, the Th1 signature cytokines, a broad role in host defense - against all intracellular microbes, including viruses, and many bacteria, fungi, and parasites. In contrast, if you consider the epidemiology of infectious diseases, it was not surprising at all. Indeed, the historical reality is that most children suffer from at most one or two severe infections. In each child, it's a different infection. One child has severe pneumococcal disease, another tuberculosis, a third diphtheria, etc. Half of children died before the age of 15 years until the second half of the 19th century, not because all microbes were virulent, but because of the enormous diversity of infectious pathogens. With this in mind, if infections are genetic, then it is not surprising that the genetic traits underlie a narrow range of infections, at least in each individual. It is also important to understand that several infections, in different individuals, can be allelic at the same human locus. The only specificity that matters, physiologically, operates at the individual level. So what explains the immunological but not pediatric surprise? Probably the fact that experimental infections in inbred mice are much more sensitive - they reveal many more phenotypes. There is a greater redundancy in outbred humans, not only for natural infections but also for iatrogenic infections caused by vaccines.

JCI: When did it become possible to try targeted therapies with recombinant cytokines in some of these patients?

Casanova: The paradox is that IFN- $\gamma$ was used before the genetic etiologies of mycobacterial diseases were deciphered. In some cases, it worked; in others, it did not work. If you look at these data with a statistical mind, you can't conclude anything, or you can conclude that IFN- $\gamma$ does not work at the "population level." However, once the genetic etiologies were discovered, everything became clear. Patients with a complete lack of receptor were not helped by IFN- $\gamma$, whereas the genetic etiologies that impaired its production but not its response benefited from it. As always, the solution came from an understanding of disease in individuals. There are only patients - diseases are words used by default. I think the asymptote in medicine is each patient defining an idiosyncratic disease. I suppose this is what people mean when referring to precision medicine; we are back, at last, to Bernard and Darwin. It essentially consists of following the trend of 200 years of human medicine, which started with a handful of conditions, counts 10,000 conditions by now, and will some day truly realize that each patient defines a specific condition, or set of conditions, obeying a specific determinism in a specific individual.

JCI: Given the explosion of tools available for genetics research, much effort has been devoted to large-scale genome-wide association studies. Yet many of your studies have begun with a single patient. What is the power you see in studying these rare individuals?

Casanova: The reason populationbased genome-wide association studies have failed, in the study of human pathogenesis, whereas patient-based, sometimes single patient-based, studies have succeeded, for infectious or other conditions, is very simple. It is exactly the same reason why Francis Galton, the professional scientist, did not discover the laws of heredity that the amateur scientist Gregor Mendel discovered. It relates to the fundamental difference between living organisms and inert matter. Whereas each copy of the JCI is identical to the other copies, each JCI reader is unique and different from other JCI readers.

This is one of Darwin's first great insights, which Ernst Mayr later referred to as "population thinking" and Archibald Garrod as "chemical individuality." Claude Bernard independently made the same observation. He was the first to use the term determinism in biology and insisted that a physiologist should stay as far as possible from population-based statistics, which not only mask our lack of understanding of the determinism but also blur its search. Indeed, both physiological and pathological determinisms operate in individuals, not in populations.

The term population genetics can be misleading, because populations do not have genes. Population-based genetic studies are wonderful tools to study the history and geography, the evolution of organisms as collections of breeding organisms - populations. These studies are much less appropriate to tackle physiological and pathological problems, because genes operate in individuals, not populations. Of course, they can be very helpful, at a small scale, in rare cases of phenotypic and genetic homogeneity, but these are in many ways more patientbased than population-based studies. The bottom line is that physiology and pathology operate at the level of single individuals.

JCI: The ASCI's honor of the Korsmeyer award comes shortly after your election to the National Academy of Medicine and the National Academy of Sciences last year. What have these accolades meant to you?

Casanova: These accolades mean a lot to me. The notion that severe infectious diseases of childhood can be caused by monogenic inborn errors of immunity which was seen two decades ago as very unlikely, if not heretic by microbiologists, immunologists, geneticists, and pediatricians, in each case for a different reason is increasingly accepted as a plausible theory, at least worth being further tested. The Korsmeyer award means that we did sufficiently well to see our work and underlying idea recognized by physician-scientists in various disciplines; it is a strong encouragement to do more and better.

\section{Sarah Jackson}

1. Ciancanelli MJ, et al. Infectious disease. Lifethreatening influenza and impaired interferon amplification in human IRF7 deficiency. Science. 2015;348(6233):448-453.

2. Jouanguy $\mathrm{E}$, et al. Interferon- $\gamma$-receptor deficiency in an infant with fatal bacille Calmette-Guérin infection. N Engl JMed. 1996;335(26):1956-1961.

3. Newport MJ, et al. A mutation in the interferon- $\gamma$-receptor gene and susceptibility to mycobacterial infection. $N$ Engl J Med. 1996;335(26):1941-1949. 